\title{
Solid methane moderators: thermodynamics and chemistry
}

\author{
O Kirichek, CR Lawson, GL Draper, DM Jenkins, \\ DJ Haynes and S Lilley
}

\section{Published version information}

Citation: O Kirichek et al. 'Solid methane moderators: thermodynamics and chemistry.' Journal of Neutron Research, vol. 22, no. 2-3 (2020): 281-286. Is in proceedings of: $23 \mathrm{rd}$ Meeting of the International Collaboration on Advanced Neutron Sources (ICANS-XXIII), Chattanooga, Tennessee, United States, 13-18 Oct 2019.

DOI: $10.3233 / J N R-190132$

The final publication is available at IOS Press through DOI above.

This version is made available in accordance with publisher policies. Please cite only the published version using the reference above. This is the citation assigned by the publisher at the time of issuing the AAM. Please check the publisher's website for any updates. 


\title{
1. Solid methane moderators: thermodynamics 2 and chemistry
}

\author{
3 O Kirichek ${ }^{\text {a, }}$, CR Lawson ${ }^{\text {a }}$, GL Draper ${ }^{\text {a }}$, DM Jenkins ${ }^{\text {a }}$, DJ Haynes ${ }^{\text {a }}$ and S Lilley ${ }^{\text {a }}$ \\ $4 \quad{ }^{a}$ ISIS Facility, STFC, Rutherford Appleton Laboratory, Harwell, Didcot, UK \\ $5 \quad$ E-mail: oleg.kirichek@stfc.ac.uk
}

Abstract. The unique properties of solid methane enable the conversion of hot, energetic neutrons into cold neutrons, with an efficiency approximately 3.5 times that of liquid hydrogen based moderators. However, practical applications of solid methane in neutron moderators turned out to be much more challenging than initially expected. Exposure of solid methane at low temperatures to neutron radiation leads to a build-up of radiolysis products in the solid methane matrix. Accumulation of defects beyond some critical number can result in a spontaneous self-accelerated recombination process, which in combination with the expansion of hydrogen built up in bulk solid methane during irradiation, was believed to be responsible for the moderator's breakdown. Here we present results of our thermodynamic model, based on the theory of thermal explosion. Our model agrees well with the test data obtained using methane moderators developed at the IPNS neutron source, based at Argonne National Laboratory and the data acquired during commissioning of the ISIS Target Station 2 solid methane moderator. We also discuss the products of radiolysis reactions generated by exposure of the condensed methane to neutron radiation. The succession of radiolysis reactions may lead to the production of long chain hydrocarbons, which can contaminate the moderator system and significantly reduce efficiency of the heat-exchanger. The possible solutions for cleaning moderators using targeted solvents are considered. In the conclusion we give some practical recommendations, based on our simulation results and operational experience.
\end{abstract}

Keywords. Neutron moderator, Solid methane, Radiolysis

\section{Introduction}

Solid methane is proven to be one of the most efficient materials for moderating neutrons. It has more than three times higher moderating efficiency than liquid hydrogen [1, 2]. However, from an engineering point of view, the supreme neutron moderating properties of solid methane are seriously challenged by inconveniences in other areas. As we found in our earlier tests, at temperatures above $60 \mathrm{~K}$ solid methane is soft and sticky [3]. Adhesion to solid methane is surprisingly the same for many different materials, such as stainless steel, aluminium alloy or even Teflon. To break a bond established between solid methane and a substrate one needs $60 \mathrm{kPa}$ of normalised force. At low temperatures methane loses its ductility and stickiness and behaves like ordinary window glass. In contrast, at high temperatures, not far from the melting point, methane behaves like a viscous non-Newtonian liquid with a dynamic viscosity similar to that of andesite lava [3].

However, the greatest inconvenience to moderator engineers is not the mechanical properties of solid methane, but the "burp" phenomenon. This was discovered by J. Carpenter and colleagues when they were testing the first solid methane moderator built for the Intense Pulsed Neutron Source at Argonne National Laboratory [1, 2]. After exposure to neutron radiation, their solid methane moderator experienced sudden, violent, warm-ups. In most cases these events were triggered by minor changes in

\footnotetext{
*Corresponding author. E-mail: oleg.kirichek@stfc.ac.uk
} 
temperature or cooling power of the cryogenic system, but occasionally, they happened spontaneously. The typical behaviour of the moderator temperature is presented in Fig. 1 from Ref. [1], where the solid methane temperature increases from $12 \mathrm{~K}$ to $40 \mathrm{~K}$ in less than 10 seconds. In a number of occurrences, the burp effect has caused serious damage to the moderator vessel. Later, E.P. Shabalin and his colleagues at IBR 2 source in Dubna studied the behaviour of solid methane in neutron radiation [4, 5]. Their results agree well with findings from the Carpenter publications $[1,2]$.

According to Carpenter's model [1] the "burp" effect is driven by the recombination of radiation defects frozen into the matrix of solid methane at cryogenic temperatures. The number of defects accumulated in bulk solid methane is proportional to the neutron flux and irradiation time. When the average distance between accumulated defects becomes small enough, the system becomes unstable and any minor fluctuation could trigger recombination, leading to the burp effect. The very first recombination reaction releases substantial heat which warms up the surrounding cryo-crystal and accelerates the recombination of nearby radiation defects. Once started the process quickly leads to a runaway reaction or thermal explosion.

In 2009 a team of ISIS engineers designed, made and commissioned a solid methane moderator for the Target Station 2 project. Based on the conclusions of Carpenter and Shabalin, the operational temperature of the moderator was set above $30 \mathrm{~K}$ in order to avoid the burp effect. However during the early stages of commissioning, the system experienced an event similar to the burp effect, which was previously thought to only occur at much lower temperatures. This event damaged the integrity of the solid methane vessel, resulting in a leak into the vacuum space.

To investigate this unexpected behaviour, the ISIS team established a small collaboration project with Prof Savchenko's group from Verkin Institute for Low Temperature Physics and Engineering in Kharkov, Ukraine. In Savchenko's experiments a thin solid methane layer was irradiated at a low temperature of 4 $\mathrm{K}$. The temperature of the substrate was then slowly increased whilst recording a number of parameters. The main conclusion of the experiments was that the recombination of radiation defects happens in two stages [6, 7]. It was assumed that the first stage, occurring at low temperatures between $10 \mathrm{~K}$ to $30 \mathrm{~K}$, is driven by recombination of light species such as atomic hydrogen, protons and electrons. The second stage takes place at higher temperatures between $40 \mathrm{~K}$ and $60 \mathrm{~K}$, and mostly involves heavier radiation defects like $\mathrm{CH}_{3}$ radicals, ions of methane and ethane.

\section{Model}

Following the conclusions of Savchenko's experiments, we introduced changes into model based on Carpenter's approach [1]. We implemented two different types of radiation defects, with different recombination rates and thermal activation energies [8]. The process of accumulation and recombination of radiolysis defects can be described by the equations:

$$
\begin{gathered}
\frac{d N_{1,2}(t)}{d t}=R_{1,2}(t)-K_{1,2}(T) N_{1,2}^{2}(t) \\
M C(T) \frac{d T}{d t}=P(t)+\varepsilon_{1} K_{1}(T) N_{1}^{2}(t)+\varepsilon_{2} K_{2}(T) N_{2}^{2}(t)-A H(T)\left(T(t)-T_{c o o l}(t)\right) \\
K_{1,2}(T)=K_{o 1,2} e^{-\Delta E_{1,2} / k_{B} T}
\end{gathered}
$$

Here, $T$ is temperature, $N_{l}$ is the number of defects recombining around $20 \mathrm{~K}$ and $N_{2}$ is the number of defects recombining at around $50 \mathrm{~K}$. Equation (1) is the same for both species, so for simplicity we use $N_{l, 2}$ where index 1 and index 2 are for lower and higher temperatures of recombination respectively. $R_{l, 2}$ is the defect production rate for each species. In our model we assume that the production rate is the same $R_{I} \approx R_{2}$ for both species. $M$ is the mass of the system, $C$ is heat capacity of solid methane, $H$ is the thermal 
conductivity, $A$ is the area available for heat-exchange between the system and coolant, $T_{\text {cool }}$ is the coolant temperature and $P$ is the heating power provided by the external source.

Equation (3) gives radiation defect recombination rate coefficients $K_{1,2}$ for each species, where $K_{01,2}$ are the recombination rates at infinite temperature for each species. $\Delta E_{1,2}$ are the activation energies for defect diffusion. We have used the activation energy of dislocation motion in solid methane at different temperatures [9] as an estimation for choosing $\Delta E_{1} / k_{B}=108.6 \mathrm{~K}$ and $\Delta E_{2} / k_{B}=235 \mathrm{~K}$. The differential equations $(1-3)$ are inherently non-linear and so we solve them numerically in order to produce a simulation of the system's behavior under different parameters. Starting at some known temperature, with a fixed defect production rate $R_{1,2}$, we calculate the new number of defects $N_{l, 2}$ from equation (1) over our suitable chosen time step $d t$. Equation (2) shows the balance of power into, and out from, the system. The process then loops over the chosen length of time.

The isochoric pressure derivative equation, also added to the model [8], allowed us to obtain the pressure change in the moderator vessel as a function of time. This approach assumes infinite rigidity of the moderator vessel's walls. When the temperature of the vessel rapidly increases, the thermal expansion of solid methane creates a pressure on the vessel walls. If the pressure rises above the maximum operational pressure, defined by the engineering design of the vessel, the integrity of the system could be compromised.

\section{Discussion}

The comparison of our model with experimental results published in Carpenter's paper [1] are presented in Figure 1. Here we show the output from our model, calculating both temperature (blue line) and pressure (red line). The results of our simulation are in good agreement with Carpenter's data. The calculated peak pressure in the vessel reaches 15 bar.

In Fig. 2 we also present a spontaneous event which occurs in the model, with parameters comparable to the IPNS moderator after exposure to the neutron radiation for more than 2.5 days [1]. A similar occurrence has been described in the text of Carpenter's paper [1], however it occurred after 21 days of irradiation at $9 \mathrm{~K}$. As one can see, the temperature of the vessel exceeds the melting point of solid methane, whilst the pressure reaches values greater than 40 bar, far above the maximum operational pressure of the moderator vessel. This model outcome agrees with the description of events where the moderator vessel has been damaged as a result of the burp effect [1].

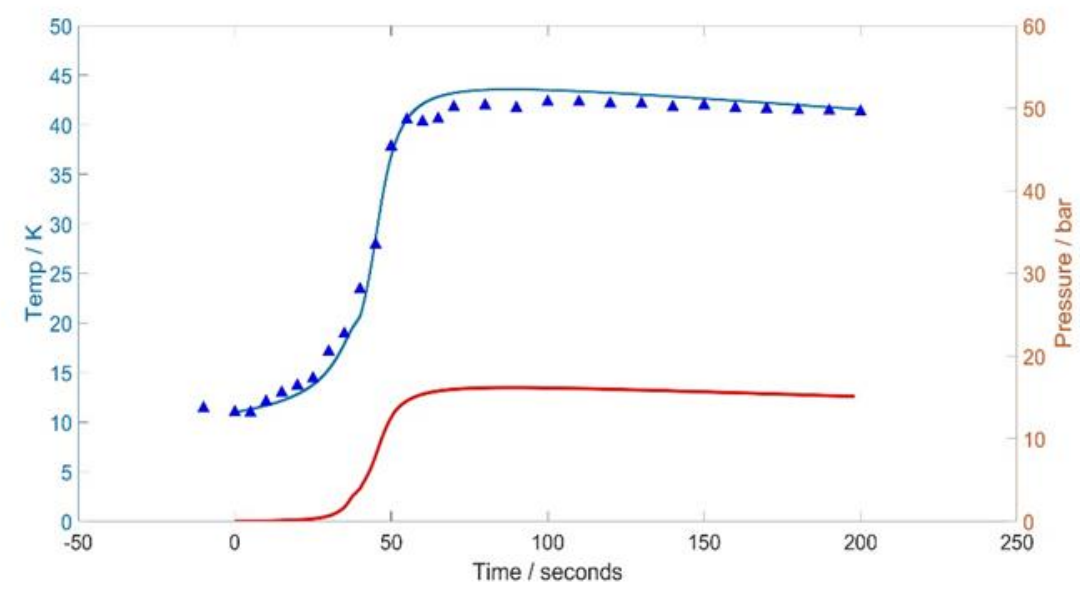


Fig. 1. Data (dots) published in [1] and the results of our simulations showing both temperature (blue line) and pressure (red line). The results of our simulation are in good agreement with [1]. The calculated peak pressure in the vessel reaches 15 bar.

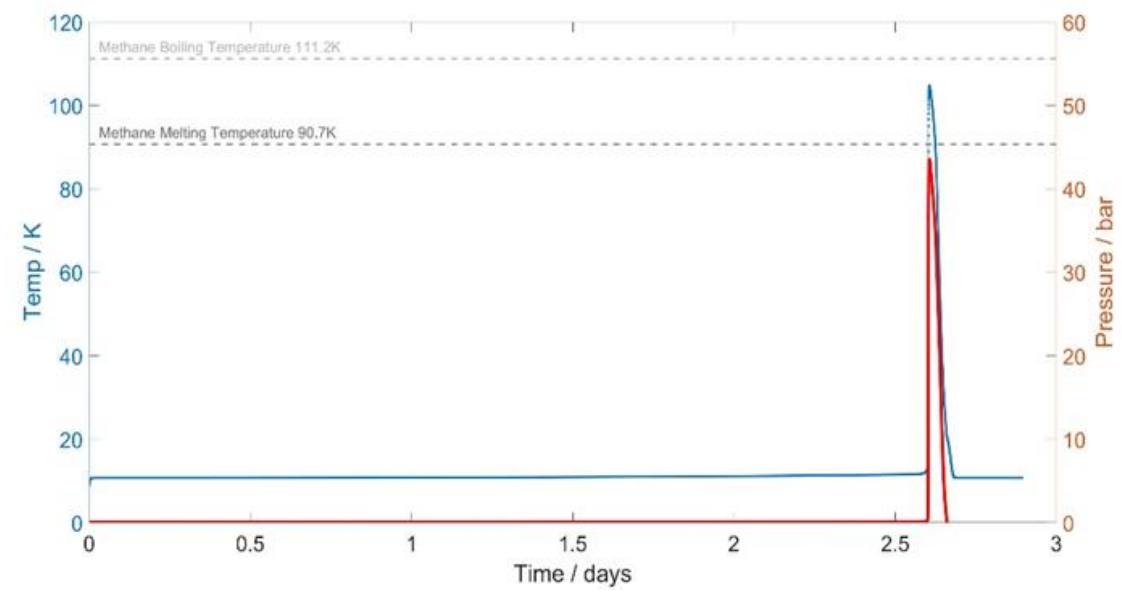

Fig. 2. The simulation of a spontaneous event described in [1] showing both temperature (blue line) and pressure (red line). The temperature of the vessel exceeds the melting point of solid methane, whilst the pressure reaches values greater than 40 bar, far above the maximum operational pressure of the moderator vessel.

The results of simulations run with parameters relevant to the test conditions of the first prototype of the ISIS TS2 solid methane moderator also, showed very good agreement with the test data. Demonstrating a peak pressure in excess of the designed maximum operational pressure of the moderator vessel. These simulations clearly explain the cause of the vacuum leak experienced during the test of the first moderator prototype. The pressure obtained in our simulations significantly exceeds the maximum pressure defined in the vessel's engineering design.

After detecting a leak in the ISIS solid methane moderator vessel mentioned above, the team of ISIS engineers made three important decisions. Firstly it was decided to increase the moderator vessel wall thickness threefold, thereby increasing the maximum operational pressure of the moderator vessel to 30 bar, so its maximum operational pressure exceeds the peak pressure achieved during the moderator warm up. Secondly, the operational temperature was increased to $47 \mathrm{~K}$. That allowed significant reduction in the accumulation of "heavy" radiolysis species, due to the higher recombination rate. Thirdly the time between complete methane changes was reduced to 19 hours, which also reduced the amount of accumulated radiolysis defects. Since implementation of these changes the ISIS solid methane moderator has never experienced problems with leaks or damage to the moderator vessel integrity. It is worth mentioning though that neither the moderator's design, nor operational procedures have been optimized.

With these modified operational procedures, it was necessary to manage the frequent methane changes automatically. Thanks to the efforts of the Control Section of the ISIS Target and Moderator group, the whole process has been completely automated by implementation of a PLC based control system, thus releasing significant technical resources [14]. 




Fig. 3. Summary of solid methane radiolysis reactions that result in production of polymer mixture. Left: mechanism for a radical driven reaction chain. Right: suggested mechanism for the ion-molecule chain reaction described in [11].

However another problem revealed itself only at a much longer time-scale. After approximately 60 days of operation the efficiency of the moderator heat-exchanger started to rapidly deteriorate and after a few days made moderator operations impossible. The reason for this deterioration was the production of large hydrocarbon molecules as a result of methane radiolysis. Neutron radiation creates favourable conditions for synthesising polymer mixtures, as shown in Figure 3, which precipitate and accumulate at the heat exchanger's aluminium surface. The thermal conductivity of polymer films is particularly low, significantly reducing the efficiency of the heat-exchanger. The radiation chemistry of solid methane has received some attention over the past 50 years [10 - 14]. Reactions from the literature suggest two possible mechanisms for the formation of a polymer film on the heat exchanger of the ISIS moderator.

The first mechanism is driven by the same radical recombination reactions which cause the burp phenomenon. Radiolysis defects such as $\mathrm{CH}_{3}$, which become mobile during the controlled warm-up of the ISIS solid methane moderator, may react with one another to form heavier molecules such as ethane. Since the solid methane moderator is only heated to $155 \mathrm{~K}$, and the boiling point of most hydrocarbons other than methane is above this temperature, the heavy molecules will remain in the moderator even when the methane is replaced. These molecules build up and may undergo radiolysis themselves, creating, for example, the ethyl radical. With each cycle of the moderator, more heavy hydrocarbons build up, with the products growing in length until a mixture of long-chain polymer is left on the heat exchanger surface.

The second possibility is a chain reaction initiated by a positively charged product of methane radiolysis, such as $\mathrm{CH}_{3}+$. Although the exact mechanism for this reaction is uncertain, Davis et al. reported formation of a polymer containing an average of 20 carbon atoms [11]. While the polymer yield 
was found to depend on accumulated dose, polymer chain length was independent of both dose and dose rate, pointing to a chain reaction of some kind. The authors point out that a high degree of branching may be expected in the polymer produced by this mechanism [10].

Regenerating the moderator's exchanger is possible by cleaning with a strong solvent such as methyl ethyl ketone (MEK). However, MEK is highly flammable and causes irritation upon exposure to fumes, so a gentler cleaning solvent is sought for routine cleaning. Several proprietary acetone-based solvent mixtures are available, each boasting lower toxicity than MEK while maintaining its excellent solvation properties. However, to choose a cleaning solvent to target the ISIS solid methane moderator, analysis of the radiolysis products is necessary.

\section{Conclusion}

In conclusion, our model (based on Carpenter's approach using Arrhenius equations) demonstrates good agreement with the IPNS test data and results from ISIS TS2 solid methane moderator commissioning. It also explains the mechanism behind damage to the solid methane moderator vessel during thermal explosion occurrences. We also review possible radiolysis processes that lead to the production of polymer mixtures, contaminating the moderator vessel heat exchanger, and suggested possible solutions to this problem.

We would like to offer some practical recommendations based on our simulations results and operational experience:

- $\quad$ Solid methane moderators can be comfortably operated above $47 \mathrm{~K}$.

- There is an optimal compromise between neutron flux, the moderator vessel's wall thickness and time between methane changes.

- A small empty space left in the moderator during methane changes, may help to reduce pressure on the vessel walls. Solid methane behaves like a viscous liquid above $60 \mathrm{~K}$. This small space would allow excess methane, near to the wall, to be squeezed out and therefore release the pressure within the moderator.

- Usage of a targeted solvent(s) for vessel cleaning may extend the moderator's life-span. Improvement to the Health \& Safety aspects of moderator cleaning may be another benefit.

- Solid methane moderator operations could be highly automated, liberating valuable technical resources.

\section{Acknowledgements}

We would like to thank Zoe Bowden, Matt Fletcher, Dennis Cowdery, Steve Wakefield, Andy Church, Rajesh Gupta, Sean Higgins, Richard Down, Rob Major, Chris Goodway, Rob Done, Mike Dudman and all others involved in the "methane" project. This project has received funding from the European Union's Horizon 2020 research and innovation programme under the Marie Sklodowska-Curie grant agreement No 645660

\section{References}

1. J. Carpenter, Thermally activated release of stored chemical energy in cryogenic media, Nature 330 (1987), 358. 
2. Scott, T. L., Carpenter, J. M., and Miller, M. E. "The development of solid methane neutron moderators at the Intense Pulsed Neutron Source facility of Argonne National Laboratory," Proceedings of the International Workshop on Cold Moderators for Pulsed Neutron Sources, 1997, pp 299-304.

3. O. Kirichek, A.J. Church, M.G. Thomas, D. Cowdery, S.D. Higgins, M.P. Dudman, Z.A. Bowden, Adhesion, plasticity and other peculiar properties of solid methane, Cryogenics 52 (2012), 325.

4. E. P. Shabalin, On the phenomenon of the fast release of energy in irradiated solid methane, JINR Commun. (1995), E1795-142.

5. E. Kulagin, S. Kulikov, V. Melikhov, E. Shabalin, Radiation effects in cold moderator materials: Experimental study of accumulation and release of chemical energy, Nucl. Instr. and Meth. B 215 (2004), 181.

6. E.V. Savchenko, O. Kirichek, C.R. Lawson, I.V. Khyzhniy, S.A. Uyutnov, M.A. Bludov, Relaxation processes in solid methane pre-irradiated with an electron beam, Nucl. Instr. and Meth. B 433 (2018), 23-27.

7. O. Kirichek, E.V. Savchenko, C.R. Lawson, I.V. Khyzhniy, D.M. Jenkins, S.A. Uyutnov, M.A. Bludov, D.J. Haynes, Recombination of radiation defects in solid methane: neutron sources and cryo-volcanism on celestial bodies, Journal of Physics: Conf. Series 969 (2018), 012006.

8. O. Kirichek, C.R. Lawson, D.M. Jenkins, C.J.T. Ridley, D.J. Haynes, Solid methane in neutron radiation: Cryogenic moderators and cometary cryo volcanism, Cryogenics $\mathbf{8 8}$ (2017), 101-105.

9. A. I. Prokhvatilov Plasticity and Elasticity of Cryocrystals (New York: Begell House Inc.) 118-132 (2001)

10. D.R. Davis, W.F. Libby, Positive-ion chemistry: high yields of heavy hydrocarbons from solid methane by ionizing radiation, Science 144 (1964), 991.

11. D.R. Davis, W.F. Libby, W.G. Meinschein, Chemistry of positive ions. VI. Positive-ion chemistry in solid methane, $J$. Chem. Phys. 45 (1966), 4481.

12. B.M. Jones, R.I. Kaiser, Application of reflectron time-of-flight mass spectroscopy in the analysis of astrophysically relevant ices exposed to ionization radiation: methane (CH4) and D4-methane (CD4) as a case study, J. Phys. Chem. Lett. 4 (2013), $1965-1971$

13. B. Smaller, M. S. Matheson, Paramagnetic species produced buy gamma irradiation of organic compounds, J. Chem. Phys., 39 (1958), 1169.

14. P. Ausloos, R. E. Rebbert, S. G. Lias, Direct and inert-gas-sensitized radiolysis and photolysis of methane in the solid phase, J. Chem. Phys., 42 (1965), 540.

15. R.K. Gupta, Solid methane moderator PLC based control system, ISIS Internal Technical Report, (2016) 